\title{
Genetic discontinuity associated with an environmentally induced barrier to gene exchange in the marine snail Littorina saxatilis
}

\author{
Jaime Piñeira, Humberto Quesada, Emilio Rolán-Alvarez, Armando Caballero* \\ Departamento de Bioquímica, Genética e Inmunología, Facultad de Biología, Universidad de Vigo, 36310 Vigo, Spain
}

\begin{abstract}
The geographical genetic structure of 7 populations of the intertidal marine snail Littorina saxatilis with limited dispersal was studied in NW Spain using 3 classes of genetic markers: 840 amplified fragment length polymorphism (AFLP) loci, 3 microsatellites, and 1 morphological trait (shell size). A sharp genetic division at Cape Finisterre, as evidenced by all 3 markers, delimits 2 regions. This genetic discontinuity coincides with an abrupt change in plankton composition, climate and oceanographic characteristics, suggesting that contemporary influences on gene flow associated with the presence of an ecological barrier maintain the genetic subdivision in NW Spain. However, the genetic structure also accords to some extent with an isolation-by-distance model despite the heterogeneous gene flow due to this ecological barrier. Microsatellites and shell size showed a strong and significant covariation in pairwise differences $\left(F_{\mathrm{ST}}\right.$ and $\left.Q_{\mathrm{ST}}\right)$ among populations, but no correlation was found between these markers and AFLPs. Similarly, both microsatellites and shell size showed a higher differentiation between the 2 regions than within each region, but this pattern was reversed for AFLPs. Differences in genomic sampling error and homoplasy could account for the discrepancy between different kinds of markers. These results point to the possibility that other marine species may show a similar pattern of intra-specific genetic divergence in NW Spain.
\end{abstract}

KEY WORDS: Microsatellites $\cdot$ AFLPs $\cdot$ Quantitative traits $\cdot$ Gene flow $\cdot Q_{\mathrm{ST}} \cdot F_{\mathrm{ST}} \cdot$ Isolation by distance $\cdot$ Ecological barrier

Resale or republication not permitted without written consent of the publisher

\section{INTRODUCTION}

Understanding the processes by which populations become genetically distinct and adapt to their local environments is a central topic in evolutionary biology (Lenormand 2002). Theoretical studies show that genetic drift and local adaptation are counteracted by the homogenizing effects of gene flow (Slatkin 1987). Consequently, species with low dispersal capabilities are more likely to experience local adaptation and genetic divergence due to natural selection than species characterized by large dispersal rates (Sotka 2005). The interpretation of patterns of genetic differentiation in terms of local adaptation, however, can be difficult because demographic events, such as population fragmentation, range expansions and founder events, can mimic the effect of natural selection. Nevertheless, demographic processes are expected to affect all loci in a roughly equal fashion, whereas selection will affect only some regions of the genome. An additional difficulty in the marine environment is that barriers to gene flow are hard to discern and rarely absolute. Oceanographic features, such as biogeographic boundaries and patterns of coastal or estuarine circulation, are some of the processes by which gene flow could be limited. However, the role of barriers as a mechanism for partitioning genetic variation in the marine environment remains insufficiently understood (Avise 2004).

Natural populations of the marine snail Littorina saxatilis provide an excellent model system to assess the interplay between gene flow, genetic drift and 
local adaptation. This snail lives in the intertidal zone along the Atlantic shores of Western Europe and North America, is ovoviviparous, feeds on the alga microflora and shows a low dispersal capability (1 to $3 \mathrm{~m} \mathrm{mo}^{-1}$ ) that typically results in a pattern of isolation-bydistance (Reid 1996). The wide geographic range of this species, coupled with apparently limited dispersal opportunities, has led to a remarkable geographic and microgeographic differentiation (Reid 1996). Indeed, the species is very polymorphic in shell traits both within and between populations and shows strong population structure for allozymes, microsatellites and mtDNA (Ward et al. 1986, Panova et al. 2006, Quesada et al. 2007). Several studies demonstrate a link between ecological adaptation, vertical and/or horizontal distribution, and microhabitat phenotypic differentiation (Rolán-Alvarez et al. 2004, Grahame et al. 2006, Quesada et al. 2007). In many areas, morphologically distinct ecotypes coexist in sympatry or allopatry separated by only a few meters, each showing a significantly higher fitness in its own microhabitat (Rolán-Alvarez et al. 1997).
The NW coast of Spain (Fig. 1a) is characterized by fjord-like estuaries that represent discrete habitats isolated from each other by barriers to dispersal and environmental tolerance (Ríos et al. 1992) and constitute a singular system to assess the importance of hydrographic factors on genetic differentiation and adaptation. Interestingly, NW Spain also appears to contain at least 2 distinct biogeographic regions (to the north and south of Cape Finisterre, Fig. 1b,c) with differences in primary production, salinity, water temperature and evaporation quantities (Fraga et al. 1982, Martínez \& Pérez 1999). During summer, a cold and nutrient-rich coastal current flows southward in the north (ACNAp, whereas a warmer and nutrient-poor current flows in the south (ACNAs) (Fraga et al. 1982, Alvarez-Salgado et al. 2003; see Fig. 1b). In September and October, the surface circulation reverses. One of the most characteristic features of the NW Spain hydrographic system is a quasi-permanent upwelling in summer in the area of Cape Finisterre which is due to the presence of a subsurface front between central water of distinct origins (ACNAp and ACNAs) (Fraga

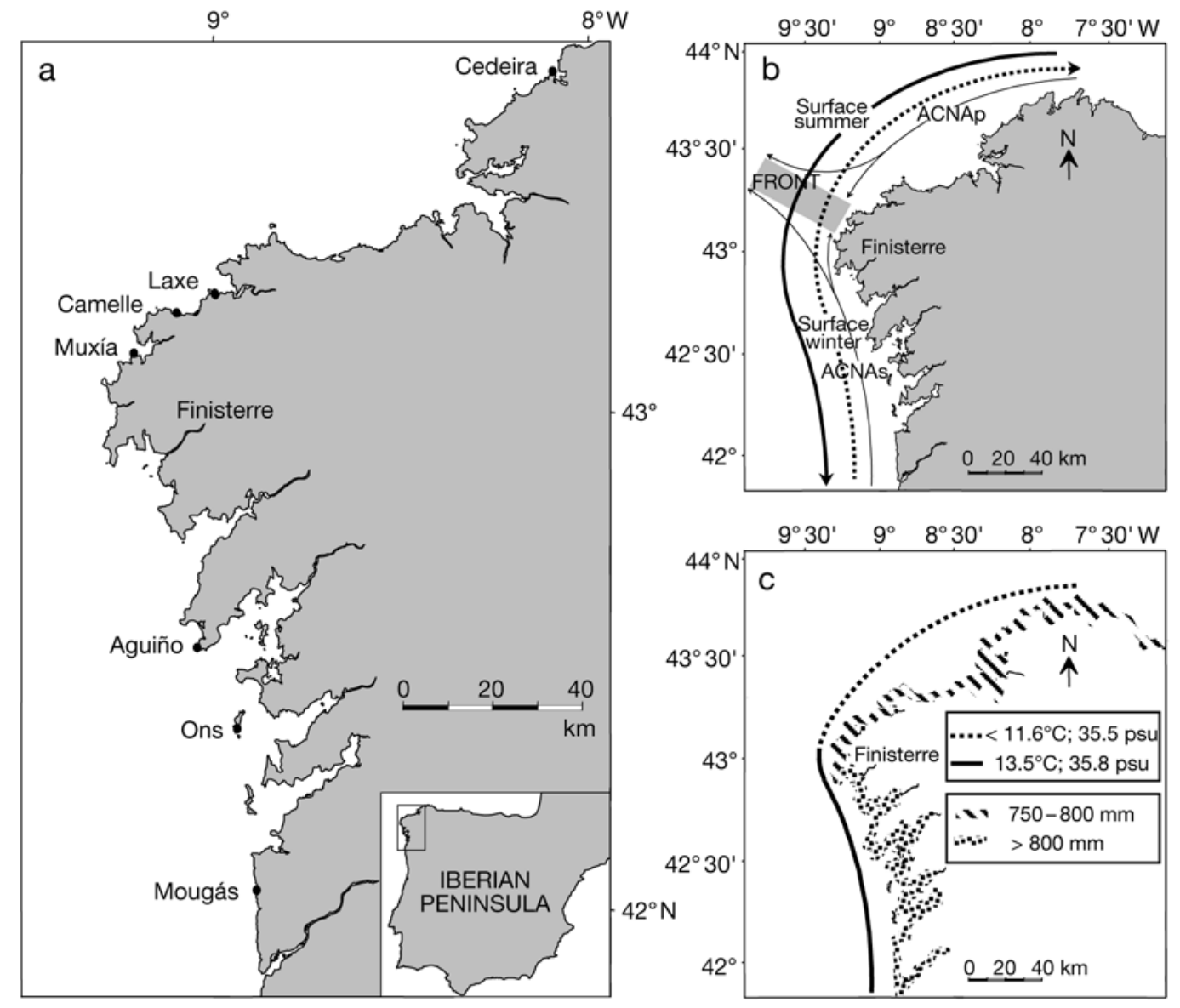

Fig. 1. (a) Sampling areas. (b) Typical marine currents and location of the subsurface front during summer (according to Fraga et al. 1982). ACNap, ACNAs: currents; see 'Introduction' for description. (c) Distribution of coastal evaporation (Martínez \& Pérez 1999) and of marine gradients in temperature and salinity (Alvarez-Salgado et al. 2003) 
et al. 1982). Although all these factors point to the importance of this biogeographic boundary for genetic differentiation, no study has yet considered its impact on patterns of population structure for any marine species.

In the present study we assessed the variation of 3 different markers (amplified fragment length polymorphisms [AFLPs], microsatellites and shell size) in Littorina saxatilis populations from NW Spain. We tested whether interpopulation genetic differentiation is gradual, as expected from the outcome of simple population genetic processes such as isolation-bydistance, or whether this genetic differentiation is discontinuous and linked to a gene flow constraint and/or adaptive processes associated with the biogeographic boundary.

\section{MATERIALS AND METHODS}

Sampling. Samples were collected June through July 2004 from 7 geographical areas of the western coast of Galicia (Fig. 1). In each area, we collected individuals from 2 sheltered localities 0.5 to $2 \mathrm{~km}$ apart (and 2 replicates per locality 10 to $20 \mathrm{~m}$ apart) to adequately represent the genetic variation of each area as a whole (20 snails per sampling site, 80 in total per area). We also extracted all developed embryos (shell height $>0.5 \mathrm{~mm}$ ) from 20 females randomly chosen from each sampling site. Four embryos per female were then chosen at random for subsequent analysis (80 embryos per sampling site, 320 in total per area). The individuals collected within a particular area were pooled in the subsequent statistical analyses.

Morphometric analysis. Shell size was measured by the centroid size, which is the square root of the sum of squared distances of landmarks (morphometric coordinate points) to their centroid (Zelditch et al. 2004). Sampled specimens were examined using a Leica MZ12 stereoscopic microscope. Color images were captured by a Leica digital ICA video camera. Embryo shell images $(\mathrm{N}=2240$ ) were analyzed using 12 landmarks (coordinate points) positioned on the digitalized shell image (see Fig. 1 in Carvajal-Rodríguez et al. 2005). Landmarks were recorded for each specimen with the program TPSDIG, while centroid size was computed with TPSRELW (http://life.bio.sunysb.edu/morph/index.html).

Molecular analysis. The shells of the adult specimens of Littorina saxatilis ( $\mathrm{N}=560)$ were broken, and the head-foot tissue was used for extracting total genomic DNA, according to a standard small-scale procedure (Wilding et al. 2001).

We assessed variation at 3 polymorphic Littorina saxatilis microsatellite loci: Lsax6CAA, $L x-12$ and $L x-18$ (Sokolov et al. 2002). PCR primers and amplification con- ditions of these 3 loci were as described previously (Sokolov et al. 2002), except that we used annealing temperatures of $61^{\circ} \mathrm{C}$ for $L s a x 6 C A A$ and $60^{\circ} \mathrm{C}$ for $L x-12$ and $L x-18$. The reverse primer was labeled with one of the fluorescent phosphoramidite dyes: 6-FAM (Lsax6CAA), HEX $(L x-12)$ or NED $(L x-18)$. The fluorescent-labeled PCR products were size-separated on an ABI PRISM 310 DNA automatic sequencer. All genotyping was performed using the software GENOTYPER 2.1 (Applied Biosystems). Scoring errors, large allele dropout and null alleles were checked employing the program MICROCHECKER (Van Oosterhout et al. 2006). GENEPOP 3.0 (Raymond \& Rousset 1995) was used to determine allele frequencies and to test the significance of departures from Hardy-Weinberg equilibrium using a Markov Chain procedure. p-values were adjusted by application of a sequential Bonferroni correction.

AFLP analysis was performed using a modified version of the procedure described by Vos et al. (1995). Briefly, the genomic DNA was double digested with EcoRI and MseI restriction enzymes. The DNA fragments were ligated with EcoRI and MseI adaptors and pre-amplified with primers carrying 1 selective nucleotide (5'-GACTGCGTACCAATTC+A-3' for ECoRI adapter and 5'GATGAGTCCTGAGTAA+C-3' for MseI adapter). The resulting product was diluted 1:20 and used for the second PCR amplification. For a single individual, selective amplification reactions were performed with 3 primer combinations: MseI + CAA with FAM-ECoR1 + ACT, MseI + CAA with HEX-EcoR1 + AAG and MseI + CAA with NED-ECoR1 + AGC. The final products were separated on an ABI PRISM 310 DNA automatic sequencer. Fragments in a range of 75 to $500 \mathrm{bp}$ were scored using GeneScan (Applied Biosystems). Allele frequencies at putative AFLP loci were estimated from presence and absence of bands using the software AFLPapp and AFLPsurv 1.0 (Vekemans et al. 2002).

Analysis of genetic differentiation. We used $F_{\mathrm{ST}}$ (microsatellites and AFLPs) or $Q_{\mathrm{ST}}$ (shell size) statistics to assess genetic differentiation among pairwise comparisons of samples. To estimate $Q_{\mathrm{ST}}$, we assumed that the chosen embryos of each female were full sibs (Conde-Padín et al. 2007). Newkirk \& Doyle (1975) demonstrated that this is a valid assumption in Littorina saxatilis after scoring 12 families of embryos for an esterase locus with 4 alleles.

Thus, $Q_{\mathrm{ST}}$ can be estimated as $Q_{\mathrm{ST}}=V_{\mathrm{b}, \text { pop }} /\left(V_{\mathrm{b}, \text { pop }}+\right.$ $\left.2 V_{\mathrm{w}, \text { pop }}\right)$, where $V_{\mathrm{b} \text {,pop }}$ and $V_{\mathrm{w} \text {,pop }}$ are the estimates of between-population and within-population genetic components of variance, respectively, the latter being equal to twice the variance among families.

The $Q_{\mathrm{ST}}$ estimates were obtained with the software MODICOS (Carvajal-Rodríguez \& Rodríguez 2005), whereas the $F_{\mathrm{ST}}$ estimates were calculated using the program GENEPOP 3.1c (Raymond \& Rousset 1995). 
We carried out a 2-level hierarchical partition of genetic differentiation (biogeographic regions and areas within regions) using an analysis of molecular variance (AMOVA) approach as implemented in ARLEQUIN (Schneider et al. 2000). For this analysis, the data were rearranged in different ways to analyze different pieces of information. At a macrogeographical level, $R$ estimates the genetic differentiation among biogeographic regions (north and south of Finisterre) after grouping areas within regions, whereas $A(R)$ estimates the genetic differentiation among areas within regions (after grouping sampling sites within each area). The statistical significance of the genetic differentiation within each partition was determined via 10000 permutations using ARLEQUIN.

Isolation-by-distance over the distribution area was assessed using the correlation of $F_{\mathrm{ST}} /\left(1-F_{\mathrm{ST}}\right)$ or $Q_{\mathrm{ST}} /\left(1-Q_{\mathrm{ST}}\right)$ against the logarithm of geographical distance. This model was evaluated using Mantel's test as implemented in the program ZT (Bonnet \& van der Peer 2002) via 10000 randomizations. Mantel tests were also performed to assess the correlation between pairwise $F_{\mathrm{ST}}$ and $Q_{\mathrm{ST}}$ estimates among samples.

We used nonmetric multidimensional scaling (MDS) (Kruskal \& Wish 1978) to visualize patterns in the relationships among the samples in terms of the $F_{\mathrm{ST}}$ and $Q_{\mathrm{ST}}$ statistics. The relative distances among samples in these ordination plots indicate the relative genetic differentiation among Littorina populations, with plots having a stress value less than 0.20 providing interpretable information concerning interpopulation relationships (e.g. Clarke 1993). The statistical significance of the differences among clusters identified by the MDS analysis was assessed a posteriori using a randomized ANOVA test (Peres-Neto \& Olden 2001). For this analysis, the coordinates on Dimension 1 from each separate cluster were randomized 10000 times, and the probability of getting an $F$-value larger than the sampled $F$ was estimated using the program ANOVA (available at: www.webs.uvigo.es/c03/webc 03/XENETICA/XB2/software.htm).

Neighbor-joining clustering was performed with PHYLIP (Felsenstein 2004). Nei's genetic distance (Nei 1972) was used for microsatellite and AFLP data, whereas $Q_{\mathrm{ST}}$ was used for shell size. The level of support of the nodes for microsatellite and AFLP data was assessed by 1000 bootstrap replicates.

\section{RESULTS}

A total of 560 individuals, representing all 7 geographical areas, were scored successfully for the 3 microsatellite and 840 AFLP loci assayed in the present study. Allele frequencies at each locus for each population are available from the authors of the current study on request. Heterozygosity levels were higher for microsatellites (mean heterozygosity $=0.837$ ) than for AFLPs (mean heterozygosity $=0.313$ ), as expected from the greater allele numbers in microsatellites. Levels of genetic variability were similar across all areas for microsatellites and AFLPs, although Cedeira exhibited a slightly higher number of alleles for microsatellites (Table 1). Tests for Hardy-Weinberg equilibrium showed a consistent trend for a deficiency of heterozygotes in microsatellite loci after sequential Bonferroni correction (Table 1). Results did not change qualitatively when considering unpooled samples for each area (Table 2) or after correcting the microsatellite data for the presence of null alleles or allele dropouts. Hence, the original microsatellite data were used. Separate estimates of AFLP genetic diversity were obtained assuming both Hardy-Weinberg equilibrium and a deviation from equilibrium equal to the average deficit of heterozygotes observed in the microsatellite loci $\left(F_{\mathrm{IS}}=\right.$ 0.245). Both methods gave roughly similar AFLP diversity estimates (Table 1).

Table 1. Microsatellite and amplified fragment length polymorphism (AFLP) variation in each Littorina saxatilis geographical area. $H_{\mathrm{e}}$ : Nei's unbiased genetic diversity (expected heterozygosity); na: number of alleles; $F_{\text {IS }}$ : deviations from Hardy-Weinberg equilibrium; PL: proportion of polymorphic loci. ${ }^{*}$ Significant deviation from the Hardy-Weinberg expectations after sequential Bonferroni correction

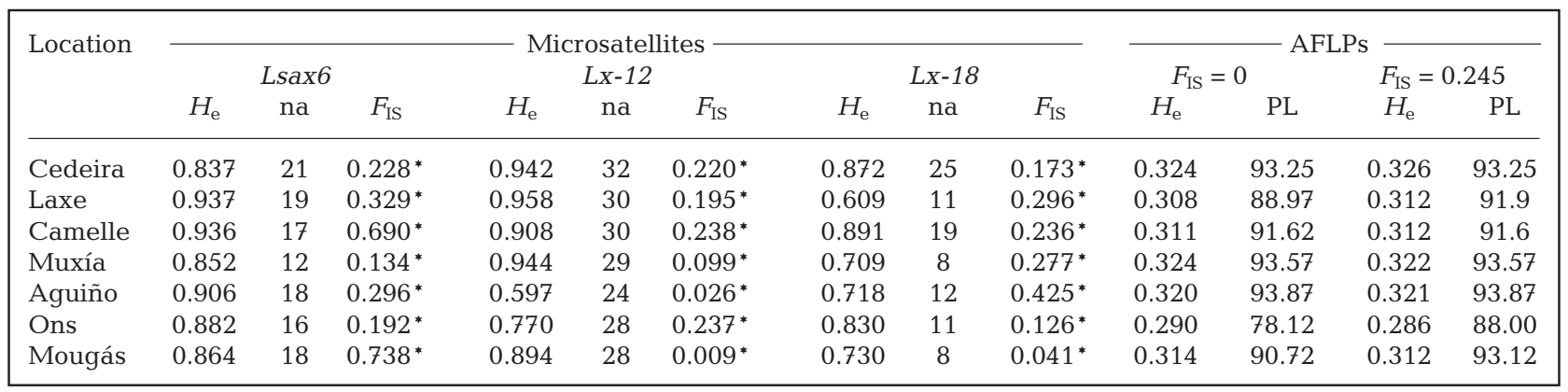


Table 2. Littorina saxatilis. Weir and Cockerham estimates of deviations from Hardy-Weinberg equilibrium per locality for microsatellite loci. $H_{\mathrm{e}}$ : Nei's unbiased genetic diversity (expected heterozygosity); $H_{\mathrm{o}}$ : observed heterozygosity; $F_{\mathrm{IS}}$ : deviations from Hardy-Weinberg equilibrium. * Significant deviation from Hardy-Weinberg expectations after sequential Bonferroni correction

\begin{tabular}{|c|c|c|c|c|c|c|c|c|c|}
\hline \multirow{2}{*}{ Location } & \multicolumn{3}{|c|}{ Lsax6 } & \multicolumn{3}{|c|}{ Lsax-12 } & \multicolumn{3}{|c|}{ Lx-18 } \\
\hline & $H_{\mathrm{e}}$ & $H_{\mathrm{o}}$ & $F_{\mathrm{IS}}$ & $H_{\mathrm{e}}$ & $H_{\mathrm{o}}$ & $F_{\mathrm{IS}}$ & $H_{\mathrm{e}}$ & $H_{\mathrm{o}}$ & $F_{\mathrm{IS}}$ \\
\hline Cedeira1 & 35.545 & 32 & $0.100^{*}$ & 32.794 & 33 & -0.006 & 27.633 & 26 & 0.059 \\
\hline Cedeira2 & 22.288 & 18 & $0.195^{*}$ & 32.000 & 24 & $0.252^{*}$ & 35.870 & 31 & $0.137^{*}$ \\
\hline Laxe1 & 23.941 & 21 & 0.125 & 27.135 & 30 & -0.107 & 18.489 & 21 & $-0.139^{*}$ \\
\hline Laxe2 & 25.618 & 22 & 0.143 & 33.087 & 22 & $0.338^{*}$ & 17.661 & 8 & $0.550^{*}$ \\
\hline Camelle1 & 16.000 & 12 & $0.255^{*}$ & 26.523 & 30 & -0.133 & 29.985 & 31 & $-0.034^{*}$ \\
\hline Camelle2 & 5.272 & 5 & 0.056 & 31.661 & 22 & $0.308^{*}$ & 27.355 & 19 & $0.309^{*}$ \\
\hline Muxia1 & 31.146 & 32 & -0.027 & 37.708 & 35 & 0.072 & 24.873 & 36 & $-0.456^{*}$ \\
\hline Muxia2 & 27.910 & 27 & 0.033 & 37.354 & 33 & $0.117^{*}$ & 9.842 & 5 & $0.496^{*}$ \\
\hline Aguino1 & 30.046 & 30 & 0.001 & 21.683 & 24 & -0.108 & 24.377 & 31 & $-0.277^{*}$ \\
\hline Aguino2 & 25.508 & 21 & $0.179^{*}$ & 25.987 & 25 & 0.038 & 12.790 & 2 & $0.846^{*}$ \\
\hline Ons1 & 31.115 & 30 & 0.036 & 16.278 & 16 & 0.017 & 30.808 & 33 & $-0.072^{*}$ \\
\hline Ons2 & 26.507 & 26 & $0.019^{*}$ & 29.857 & 28 & 0.063 & 27.813 & 25 & $0.102^{*}$ \\
\hline Mougás1 & 15.424 & 12 & $0.227^{*}$ & 35.708 & 38 & -0.065 & 30.189 & 28 & $0.073^{*}$ \\
\hline Mougás2 & 9.190 & 4 & $0.576^{*}$ & 35.886 & 33 & $0.081^{*}$ & 27.417 & 28 & $-0.021^{*}$ \\
\hline
\end{tabular}

The MDS of pairwise $F_{\mathrm{ST}}$ and $Q_{\mathrm{ST}}$ estimates for the 7 geographical areas generated plots with good fit to the observed data (Fig. 2). The stress values $(s)$ were small for microsatellites $(s=0.0006)$, AFLPs $(s=0.0062)$ and shell size $(s=0.0002)$, where $s=0$ indicates a perfect fit. For all 3 data sets, the MDS revealed 2 clusters that showed a clear concordance with biogeography. Differences between areas to the north and south of Cape Finisterre, grouped in separate clusters, were statistically significant $(p<0.05)$ for each data set as determined by a randomized ANOVA test comparing the Dimension 1 coordinates from each cluster. The
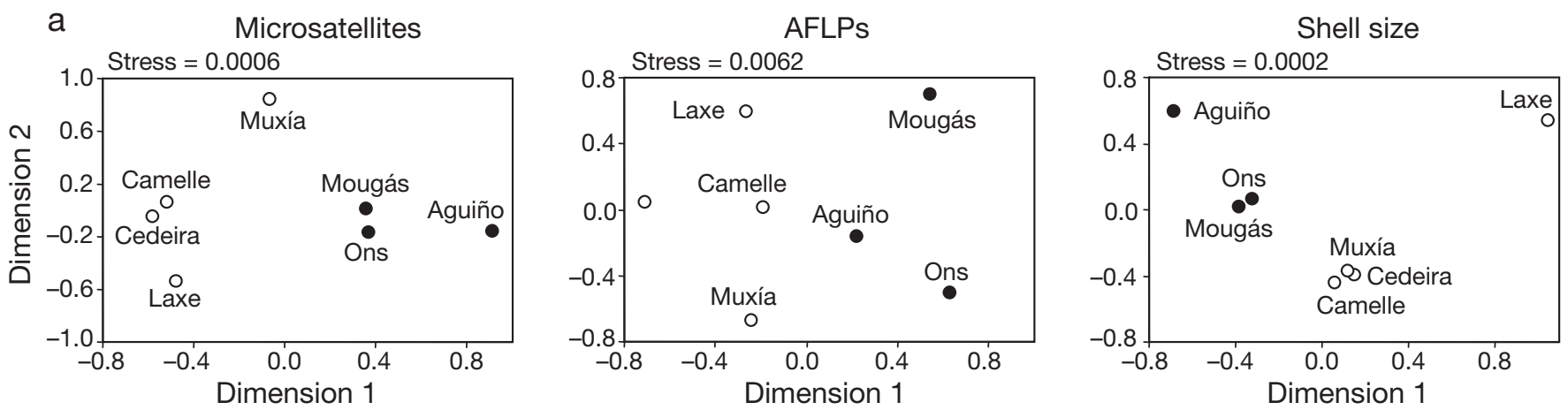

b
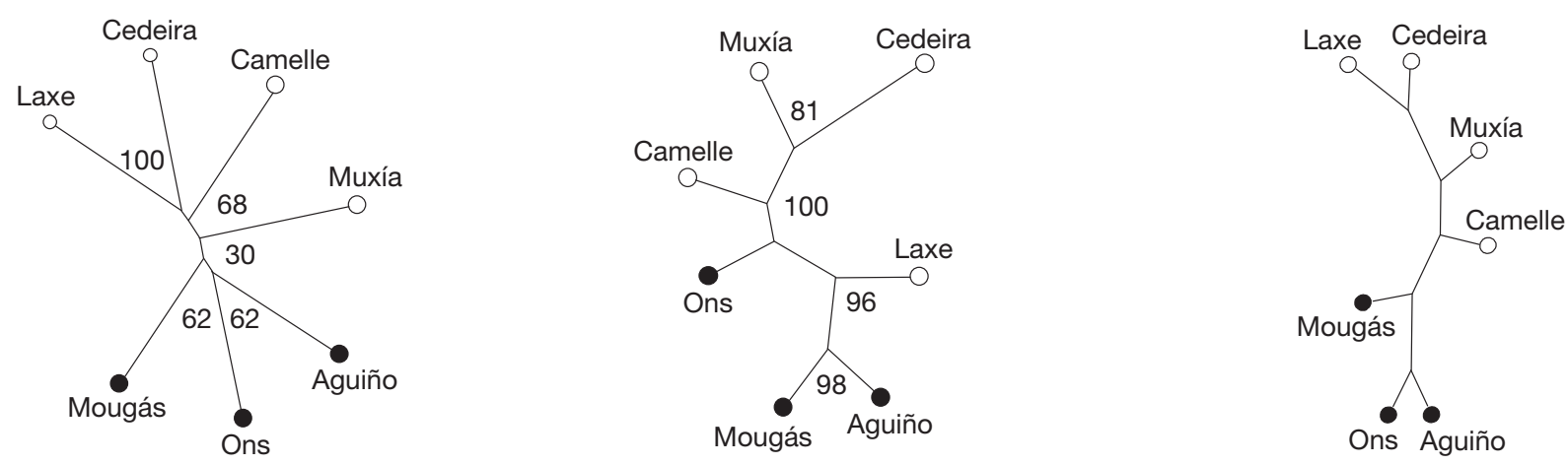

Fig. 2. Littorina saxatilis. Relationships among samples for each type of genetic marker. (a) Multidimensional scaling plots based on $F_{\mathrm{ST}}$ (microsatellites and amplified fragment length polymorphisms [AFLPs]) and $Q_{\mathrm{ST}}$ (shell size) estimates. (b) Neighborjoining clustering based on Nei's genetic distances (microsatellites and AFLPs) and $Q_{\mathrm{ST}}$ (shell size) estimates. Bootstrap values shown at the nodes. Southern $(\bullet)$ and northern $(\mathrm{O})$ areas 


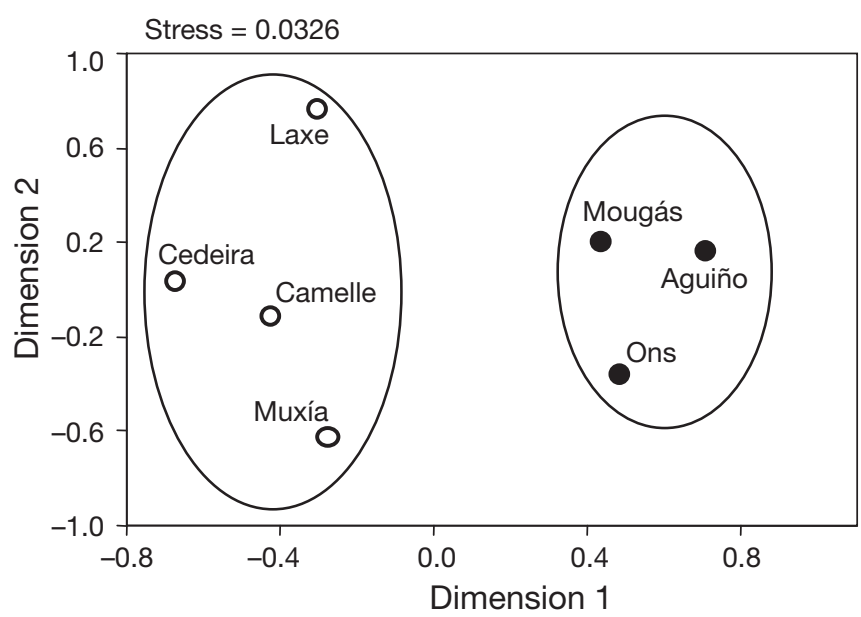

Fig. 3. Littorina saxatilis. Multidimensional scaling plot illustrating the relationships among samples combining the pairwise population differentiation estimates for microsatellites, amplified fragment length polymorphisms (AFLPs) and shell size. Southern populations (-) and northern (O) populations

neighbor-joining trees (Fig. 2) revealed essentially the same pattern as the MDS analyses, although Ons and Laxe showed a less evident grouping by biogreographical region in the AFLP data. The discrimination between samples from each biogeographic region was substantially increased when the multidimensional scaling combined the $F_{\mathrm{ST}}$ and $Q_{\mathrm{ST}}$ matrices for the 3 data sets (Fig. 3, $s=0.0326$ ), resulting in highly

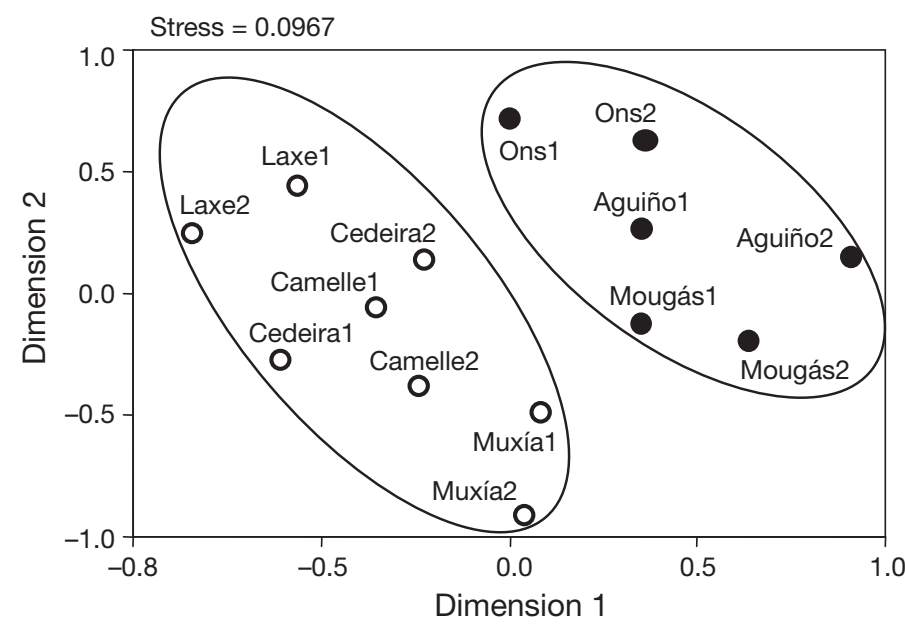

Fig. 4. Littorina saxatilis. Multidimensional scaling plot of the relationships among the localities sampled in each study area. Pairwise population differentiation estimates for microsatellites, amplified fragment length polymorphisms (AFLPs) and shell size were combined. Southern populations $(\bullet)$ and northern populations $(O)$. Differences between clusters are statistically significant $(\mathrm{p}=0.0015)$ as determined by a randomized ANOVA test comparing the Dimension 1 coordinates of each cluster significant $(p=0.018)$ differences among clusters. A similar pattern was observed when the analyses were based on unpooled samples from each area (Fig. 4).

The hierarchical analysis of the distribution of genetic variation (AMOVA) indicated that variability is significantly partitioned among areas and biogeographic regions for the 3 data sets (Table 3). Microsatellites and shell size gave similar results: generally higher differences between biogeographic regions than among areas within regions, and a lower differentiation for southern than for northern populations. However, for AFLPs the between-region variance component was at least $50 \%$ smaller than the among-area component within each region, indicating that the within-region component accounts for most of the genetic diversity. Taken overall, these results indicate that AFLPs appear to have a moderate differentiation among biogeographic regions with respect to microsatellites and shell size, perhaps as a result of a differential response to local processes within regions. Consistent with this, Mantel tests among pairwise $F_{\mathrm{ST}}$ and $Q_{\mathrm{ST}}$ estimates (Fig. 5) revealed a strong and significant correlation among microsatellites and shell size for unpooled samples $(\mathrm{r}=0.313, \mathrm{p}<0.05)$ but not between AFLPs and microsatellites $(r=0.176, p>0.05)$ or between AFLPs and shell size $(r=0.097, p>0.05)$. These results imply the AFLPs as the source of the lack of correlation.

Although the above results clearly indicate a genetic break associated with a biogeographic boundary, we could nevertheless identify an isolation-by-distance pattern. A scatterplot of the logarithm of geographical distance versus $F_{\mathrm{ST}} /\left(1-F_{\mathrm{ST}}\right)$ or $Q_{\mathrm{ST}} /\left(1-Q_{\mathrm{ST}}\right)$ illustrates an overall trend of increasing differentiation with increased geographical distance among unpooled samples for all 3 markers (Fig. 6). The results were highly significant for microsatellites $(\mathrm{r}=0.267, \mathrm{p}<0.01)$ and AFLPs ( $r=0.470, \mathrm{p}<0.001)$, whereas the trend was less clear and marginally significant for shell size $(\mathrm{r}=0.147, \mathrm{p}<0.10)$. No significant results were obtained when the tests were performed separately within northern and southern populations, which, however, showed similar trends to those observed for the total data set (not shown).

Table 3. Littorina saxatilis. Two-level hierarchical partition of genetic variability. $R$ : genetic differentiation between biogeographical regions; $A(R)$ : genetic differentiation among areas within regions. AFLPs: amplified fragment length polymorphisms. ${ }^{* * *} \mathrm{p}<0.001$

\begin{tabular}{|lccl|}
\hline Marker & \multicolumn{3}{c|}{$A(R)$} \\
\cline { 2 - 4 } & $R$ & North & \multicolumn{1}{c|}{ South } \\
\hline Microsatellites & $0.052^{* * *}$ & $0.057^{* * *}$ & $0.038^{* * *}$ \\
AFLPs & $0.022^{* * *}$ & $0.052^{* * *}$ & $0.059^{* * *}$ \\
Shell size & $0.083^{* * *}$ & $0.040^{* * *}$ & 0.002 \\
\hline
\end{tabular}



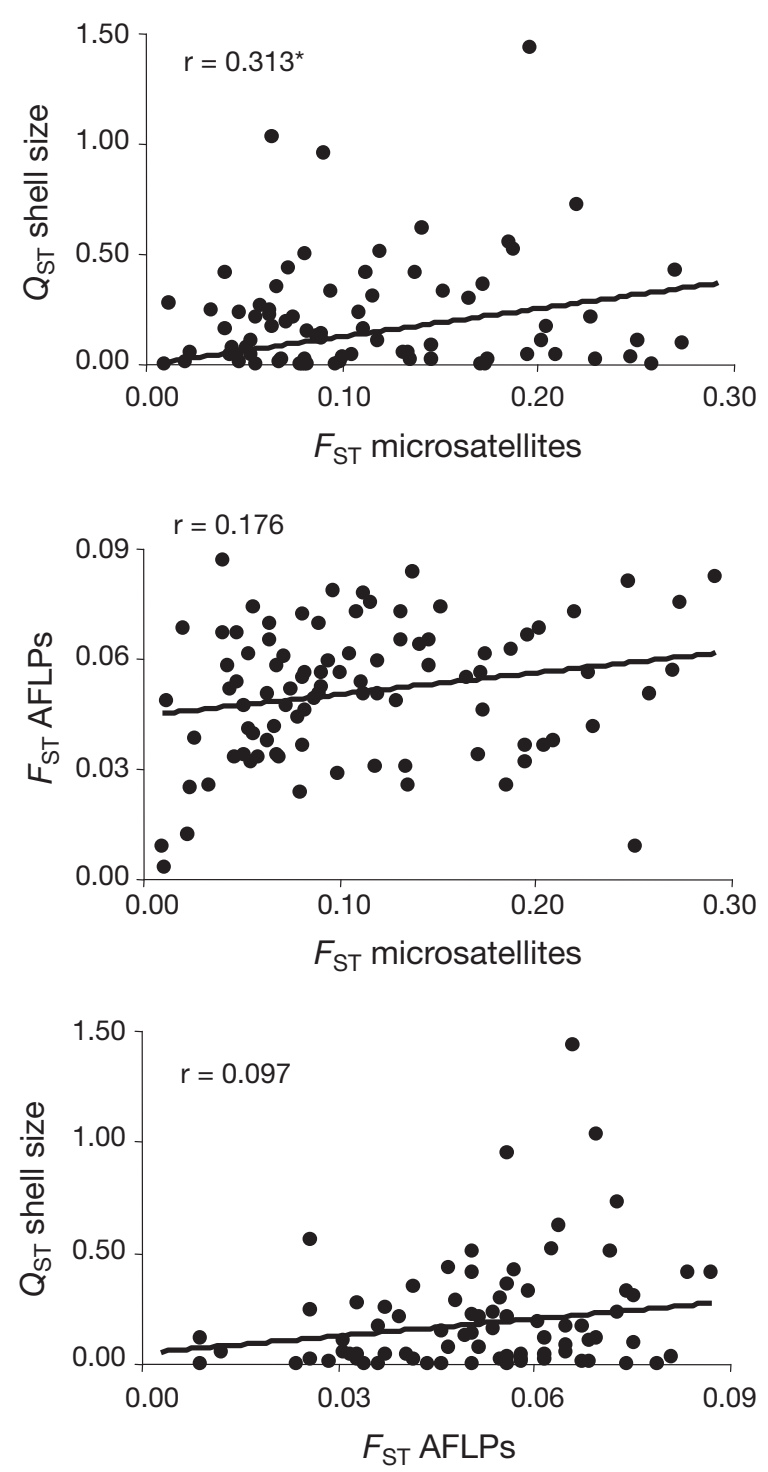

Fig. 5. Littorina saxatilis. Covariation among markers. Pairwise comparison of genetic differentiation among genetic markers. p-values were determined using a Mantel test with 10000 randomizations. ${ }^{*} \mathrm{p}<0.05$

\section{DISCUSSION}

Geographic adaptation, genetic drift and migration can all have profound effects on the genome-wide patterns of variability of a species. To assess the impact of these forces in the genome of Littorina saxatilis, we examined patterns of polymorphism and genetic differentiation using 3 different genetic markers: AFLPs, microsatellites and shell size. The data analysis presented in the present study strongly supports the hypothesis that population structuring in L. saxatilis is strongly influenced by an ecological barrier to gene flow in NW Spain. This is supported by the observation that all 3 markers
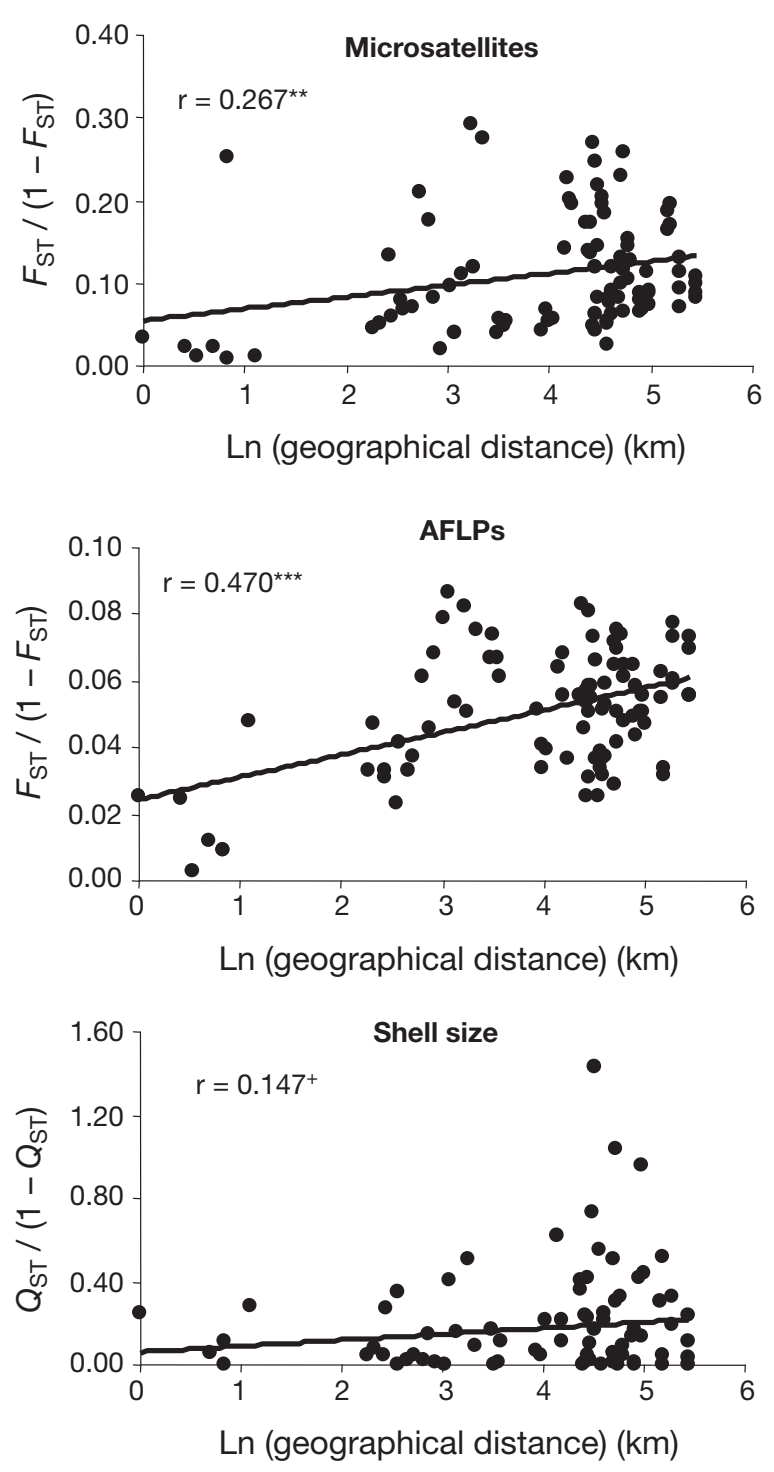

Fig. 6. Littorina saxatilis. Isolation by distance. Pairwise comparisons of genetic differentiation in different genetic markers against geographical distance. ${ }^{* *} \mathrm{p}<0.01,{ }^{* * *} \mathrm{p}<$ $0.001,{ }^{+} \mathrm{p}<0.10$

show a concordant genetic break that coincides in position with an abrupt change in climate, ecological characteristics and oceanographic circulation patterns. The combined influence of these water currents and ecological gradients is probably a major factor responsible for the position and maintenance of the genetic discontinuity. To our knowledge, the current study is the first showing a separation between northern and southern populations at Cape Finisterre, as this boundary has never before been recognized as a genetic barrier.

The heterozygote deficit observed in the present study for microsatellites is a common phenomenon in populations of marine mollusks (Zouros \& Foltz 1984) and has 
been recurrently observed in Littorina saxatilis for allozyme and microsatellite loci (Ward et al. 1986, Panova et al. 2006). Heterozygote deficits are commonly attributed to technical errors. However, MICROCHECKER showed no indication that genotyping errors affected allele scoring (e.g. allele dropouts or stuttering) at any of the microsatellites in any of the samples, although these loci might be influenced by one or more null alleles. High frequency of null alleles may complicate population genetic analyses that rely on Hardy-Weinberg equilibrium because false homozygotes would be common (e.g. de Sousa et al. 2005). Nevertheless, samples that failed to amplify were rare, thus indicating that null homozygotes were not common in our samples. Therefore, adjusting the data set with MICROCHECKER to take into account the putative presence of null alleles did not modify the results obtained. No sample failed to amplify at more than one locus, making it unlikely that poor DNA quality affected our results. Causes for a heterozygote deficit, other than from technical errors, generally include substructuring of the population sample, inbreeding or hitchhiking with a selected locus (e.g. Zouros \& Foltz 1984). Interestingly, $F_{\text {IS }}$ was found to increase across all loci from unpooled samples (avg. $F_{\mathrm{IS}}=$ 0.104, Table 2) to pooled samples (avg. $F_{\mathrm{IS}}=0.245$, Table 1). This result suggests that a likely cause of the heterozygote deficit could be a micro-spatial Wahlund effect generated by pooling samples with a cryptic population structure.

It is difficult to determine with certainty if the observed genetic break was generated in allopatry or whether these differences were generated in sympatry by natural selection. Indeed, the 2 biogeographic regions represent habitats sufficiently divergent to lead to the observed differentiation through disruptive selection. Alternatively, NW Spain is a biogeographic border where many differentiated populations could presumably meet. Under the last scenario, the most parsimonious explanation for the persistence of the genetic break is that differences between allopatrically diverged populations are being homogenized very slowly after secondary contact in NW Spain.

Several aspects of the data, however, cannot be explained by this biogeographic boundary alone. In particular, the observation of an increased genetic differentiation with geographical distance suggests an isolation-by-distance model of genetic exchange. This result is consistent with very limited migration and indicates that a pattern of isolation-by-distance may be compatible with the presence of a major ecological barrier to gene flow. Thus, whereas limited adult dispersal is likely restricting gene flow among neighboring populations at a local scale, the biogeographic boundary at Cape Finisterre appears to be the major barrier to gene flow at a macro-geographical scale.
Another striking result that apparently deviates from a simple ecological barrier scenario is that AFLP loci displayed a slightly different picture of genetic differentiation among samples from that observed for microsatellites. This distinctive variation includes a generally higher differentiation between areas than among regions and a pattern of pairwise differentiation among samples uncoupled with that observed for microsatellites. Such discrepancies among different kinds of molecular markers are often observed in comparative studies (e.g. Gaudeul et al. 2004). However, genomic sampling error rather than selection is commonly invoked to explain these discrepancies (Bierne et al. 2003). For example, if only a small number of neutral loci are compared between populations, it is likely that one or a few loci display extreme patterns of population differentiation just by neutral genetic drift. In the present study, 3 microsatellite loci are probably not enough to provide an exact view of the whole genome. Thus, in this case AFLPs could be more reliable than microsatellites, and the discrepancy between AFLPs and microsatellites could be due to the better genome coverage provided by the 840 AFLP loci. Another plausible explanation for the lack of concordance between AFLPs and microsatellite markers is that they may suffer different levels of homoplasy, which occurs when alleles identical in state are not identical by descent (Estoup et al. 2002). For both types of markers homoplasy probably increases with genetic divergence between samples, thus biasing downward the estimates of genetic differentiation (Estoup et al. 2002, Vekemans et al. 2002). Therefore, homoplasy is expected to have a greater effect on AFLPs when many loci are scored per primer combination (more than 200 in our case) due to the lack of homology of comigrating fragments (Vekemans et al. 2002). Hence, in the present study, the generally higher differentiation between areas than among regions for AFLP loci suggests higher homoplasy of AFLPs compared to microsatellites.

Genetic or ecological causes (or a combination of both) could also explain the shell size differences among populations on both sides of Cape Finisterre. It might be argued that mating asynchrony could be responsible for the different shell sizes of embryos collected among the 2 regions. According to this hypothesis, variation in shell size across redions would be the result of differences in average embryo age. This explanation, however, is unlikely since Littorina saxatilis populations from NW Spain mate and produce embryos during the entire year (Reid 1996). Consistent with this, all females examined in the present study had embryos in their embryo pouches, indicating a low frequency of non-mated females in populations from 
each region. We note that females contain embryos at different developing stages, but we only extracted developed embryos (shell height $>0.5 \mathrm{~mm}$ ) from females randomly chosen from each sampling site. This supports the view that genetically based differences are at least partially involved in the observed pattern of quantitative variation.

Genetic differentiation between subpopulations coincident in position with a biogeographic boundary has been observed in several other marine species (reviewed in Avise 2004). In many cases, local adaptation and distinct hydrographic features were proposed as causal agents in the breakdown of gene flow (e.g. Quesada et al. 1995). For example, the long-term climate and topographical changes in coastal California have been posited as a major factor influencing the evolution of marine organisms (Burton \& Lee 1994). The discovery in this area of a close concordance among these environmental changes with the phylogeographic and biogeographic patterns of marine invertebrates and fishes suggested that similar processes influenced the evolution of coastal marine taxa (Dawson 2001). Our results suggest that the observed patterns of differentiation may be maintained by environmentally induced restrictions in gene flow that allow genetic drift, and perhaps natural selection, to cause the observed genetic differentiation. Further studies on other widely distributed species will shed light on the origin of the differences detected in Littorina saxatilis. If genetic surveys of additional taxa reveal other examples of northern-southern genetic discontinuities in NW Spain, this pattern would point to a historical factor affecting many species.

Acknowledgements. We thank P. Alvariño and N. Santamaría for technical help and 3 anonymous referees for comments on the present study. This work was funded by Ministerio de Educación y Ciencia (VEM2003-20047, CGL2004-03920 BOS) and Fondos Feder, Xunta de Galicia (PGIDIT06PXIB310 247PR), and Plan Estratégico del INIA (CPE03-004-C2). J.P. was supported in his investigation by the Ministerio de Planificación y Coordinación-Chile and the Facultad de Ciencias of the Universidad Católica de la Santísima Concepción.

\section{LITERATURE CITED}

Alvarez-Salgado XA, Figueiras FG, Pérez FF, Groom S and others (2003) The Portugal coastal counter off N.W. Spain: new insights on its biogeochemical variability. Prog Oceanogr 56:281-321

Avise JC (2004) Molecular markers, natural history and evolution, 2nd edn. Sinauer, Sunderland, MA

Bierne N, Daguin C, Bonhomme F, David P, Borsa P (2003) Direct selection on allozymes is not required to explain heterogeneity among marker loci across a Mytilus hybrid zone. Mol Ecol 12:2505-2510

Bonnet E, van der Peer Y (2002) ZT: a software tool for simple and partial Mantel tests. J Stat Soft 7:1-12

Burton RS, Lee BN (1994) Nuclear and mitochondrial gene genealogies and allozyme polymorphism across a major phylogeographic break in the copepod Tigriopus californicus. Proc Natl Acad Sci USA 91:5197-5201

Carvajal-Rodríguez A, Rodríguez MG (2005) MODICOS: morphometric and distance computational software oriented for evolutionary studies. Online J Bioinformatics $6: 34-41$

Carvajal-Rodríguez A, Conde-Padín P, Rolán-Alvarez E (2005) Decomposing shell form into size and shape by geometric morphometric methods in two sympatric ecotypes of Littorina saxatilis. J Molluscan Stud 71: 313-318

Clarke KR (1993) Nonparametric multivariate analyses of changes in community structure. Aust J Ecol 18:117-143

Conde-Padín P, Grahame J, Rolán-Alvarez E (2007) Detecting shape differences in species of the Littorina saxatilis complex by morphometric analysis. J Molluscan Stud 73: $147-154$

Dawson MN (2001) Phylogeography in coastal marine animals: a solution from California? J Biogeogr 28:723-736

de Sousa SN, Finkeldey R, Gailing O (2005) Experimental verification of microsatellite null alleles in Norway spruce (Picea abies [L.] Karst.): implications for population genetic studies. Plant Mol Biol Rep 23:113-119

Estoup A, Jarne P, Cornuet JM (2002) Homoplasy and mutation model at microsatellite loci and their consequences for population genetics analysis. Mol Ecol 11:1591-1604

Felsenstein J (2004) PHYLIP (phylogeny inference package), version 3.6.5. Department of Genetics, University of Washington, Seattle, WA

Fraga F, Mouriño C, Manríquez M (1982) Las masas de agua en las costas de Galicia: junio-octubre. Res Exp Cient 10:51-77

Gaudeul M, Till-Bottraud I, Barjon F, Manel S (2004) Genetic diversity and differentiation in Eryngium alpinum L. (Apiaceae): comparison of AFLP and microsatellite markers. Heredity 92:508-518

Grahame JW, Wilding CS, Butlin RK (2006) Adaptation to a steep environmental gradient and an associated barrier to gene exchange in Littorina saxatilis. Evolution Int J Org Evolution 60:268-278

Kruskal JB, Wish M (1978) Multidimensional scaling. Sage Publications, London

Lenormand T (2002) Gene flow and the limits to natural selection. Trends Ecol Evol 17:183-189

Martínez C, Pérez A (1999) Atlas climático de Galicia. Consellería de Medio Ambiente, Centro de Información e Tecnoloxía Ambiental da Xunta de Galicia, Santiago de Compostela

Nei M (1972) Genetic distance between populations. Am Nat 106:283-292

> Newkirk GF, Doyle RW (1975) Genetic analysis of shell-shape variation in Littorina saxatilis on an environmental cline. Mar Biol 30:227-237

Panova M, Hollander J, Johannesson K (2006) Site-specific genetic divergence in parallel hybrid zones suggests nonallopatric evolution of reproductive barriers. Mol Ecol 15:4021-4031

Peres-Neto PR, Olden JD (2001) Assessing the robustness of randomization tests: examples from behavioural studies. Anim Behav 61:79-86

Quesada H, Beynon CM, Skibinski DOF (1995) A mitochondrial DNA discontinuity in the mussel Mytilus galloprovincialis Lmk: pleistocene vicariance biogeography and secondary intergradation. Mol Biol Evol 12:521-524

Quesada H, Posada D, Caballero A, Morán P, Rolán-Alvarez E (2007) Phylogenetic evidence for multiple sympatric eco- 
logical diversification in a marine snail. Evolution Int J Org Evolution 61:1600-1612

Raymond M, Rousset F (1995) Genepop (version-1.2)population genetics software for exact tests and ecumenicism. J Hered 86:248-249

Reid DG (1996) Systematics and evolution of Littorina. Ray Society, London

Ríos AF, Nombela MA, Pérez FF, Rosón G, Fraga F (1992) Calculation of runoff to an estuary. Ría de Vigo. Sci Mar 56:29-33

Rolán-Alvarez E, Johannesson K, Erlandsson J (1997) The maintenance of a cline in the marine snail Littorina saxatilis: the role of home site advantage and hybrid fitness. Evolution Int J Org Evolution 51:1838-1847

Rolán-Alvarez E, Carballo M, Galindo J, Morán P and others (2004) Nonallopatric and parallel origin of local reproductive barriers between two snail ecotypes. Mol Ecol 13: 3415-3424

Schneider S, Roessli D, Excoffier L (2000) Arlequin 2.0: a software for population genetics data analysis. Genetics and Biometry Laboratory, University of Geneva, Geneva

Slatkin M (1987). Gene flow and the geographic structure of natural populations. Science 236:787-792

Sokolov EP, Sokolova IM, Pörtner HO (2002) Polymorphic microsatellite DNA markers from the marine gastropod Littorina saxatilis. Mol Ecol Notes 2:27-29

Editorial responsibility: Otto Kinne, Oldendorf/Luhe, Germany
Sotka EE (2005) Local adaptation in host use among marine invertebrates. Ecol Lett 8:448-459

Van Oosterhout C, Weetman D, Hutchinson WF (2006) Estimation and adjustment of microsatellite null alleles in nonequilibrium populations. Mol Ecol Notes 6:255-256

Vekemans X, Beauwens T, Lemaire M, Roldán-Ruiz I (2002) Data from amplified fragment length polymorphism (AFLP) markers show indication of size homoplasy and of a relationship between degree of homoplasy and fragment size. Mol Ecol 11:139-151

Vos P, Hogers R, Bleeker M, Reijans M and others (1995) AFLP: a new technique for DNA fingerprinting. Nucleic Acids Res 23:4407-4414

Ward RD, Warwick T, Knight AJ (1986) Genetic analysis of ten polymorphic enzyme loci in Littorina saxatilis (Prosbranchia: Mollusca). Heredity 57:233-241

Wilding CS, Butlin RK, Grahame J (2001) Differential gene exchange between parapatric morphs of Littorina saxatilis detected using AFLP markers. J Evol Biol 14: 611-619

Zelditch ML, Swiderski DL, Sheets HD, Fink WL (2004) Geometric morphometrics for biologists, a primer. Elsevier, Amsterdam

Zouros E, Foltz DW (1984) Possible explanations of heterozygote deficiency in bivalve molluscs. Malacologia 25: 583-591

Submitted: May 22, 2007; Accepted: October 15, 2007

Proofs received from author(s): March 14, 2008 